Article

\title{
Extension of space syntax methods to generic urban variables
}

\author{
Roberto D'Autilia ${ }^{1, t, \ddagger}$ and Marco Spada ${ }^{2, *}$ \\ 1 Dipartimento di Matematica e Fisica, Università Roma tre; roberto.dautilia@uniroma3.it \\ 2 IRCAS - Goldmann \& Partners International Research Centre for Applied Sustainability; spada@goldmann.it \\ * Correspondence: roberto.dautilia@uniroma3.it; Tel.: +30-347-875-2989 \\ + Current address: Affiliation 3 \\ $\ddagger$ These authors contributed equally to this work.
}

\begin{abstract}
We studied generalization of a method for extending configurational studies to variables that are not exclusively geographical, in order to allow investigation of generic relationships in the built environment. We observed a number of limitations of the classical approach of configurational studies and we considered how a complex analytical method could be implemented in the study of non-topological variables, such as land use, noise pollution and financial or property rents. In order to do this we established a system of relationships based on a labeled primary graph. Categorization of the labels of the links was performed on a generic variable, specifically, the predominant use of roads. The graph was then contracted using an optimization algorithm, which we describe. The algorithm allows the network to be reduced to a more streamlined system that can better analyze the relationships between the different categories. Application of the method showed that it is faster to identify weaknesses in urban networks, and then take measures to resolve them. The case study concerns the pedestrianization of the Colosseum area in Rome.
\end{abstract}

Keywords: keyword 1; keyword 2; keyword 3 (list three to ten pertinent keywords specific to the article, yet reasonably common within the subject discipline.)

\section{Introduction}

The introduction of Space Syntax [10] in urban studies has certainly had the merit of highlighting the importance of urban topology for activities connected with the use of open spaces, and in particular for the movement of pedestrians [4]. In addition, its reformulation in terms of graph theory has opened new theoretical and use perspectives, linked to the processes of diffusion on dual graphs [2]. Shifting attention from the Euclidean geometry of urban space to its connectivity has shown that the city is a tool to connect urban phenomena: a given phenomenon, for instance the location of commercial activities, can have different effects when it manifests in differently connected spaces [6].

Among the many variables that define the behavior of a city, Space Syntax has considered almost exclusively spatial ones. Axial lines or lines of sight are the fundamental element of the model [4], variables whose topology is conditioned by the shape of the city. In graph formulation these lines are represented by graph nodes, while where they cross determines the graph's edges.

Since the choice of this variable is to some extent arbitrary, many proposals have been advanced to make it objective $[3,5,7]$. In any case the resulting graph $(V, E)$ always consists of open spaces $v \in V$ and connections between them $(u, v) \in E$ where $u, v \in V$. It is important to note that the graph represents neither the geometry nor the topology of the city, but rather the way in which the city connects the axial lines. 
On the basis of this observation we can imagine that nodes $v$ of the graph are generic urban variables, for example the amount of urban greenery, pollution levels, the income of inhabitants or even the usual Space Syntax axial lines. These quantities are naturally geolocalized, but their connection is conditioned by the structure and shape of the city. It is therefore possible to establish these connections and study how the city of green areas, income or services, and of course also the "classic" city of axial lines, are organized.

In this paper we propose a rigorous method to extend Space Syntax to generic variables. As a case study we investigate the consequences of pedestrianizing a central archaeological area in Rome (Italy).

The paper is organized as follows. In the next section we illustrate the method to obtain the generalized graph and show how, in the particular case of axial lines, the same results are obtained as with the Space Syntax approach. In section 3 we use the method to study pedestrian mobility in the central archaeological area of Rome, comparing the current situation with the layout proposed by Roma Tre University. In section 4 we show the results of the proposed technique, and how the method makes it possible to examine an urban project using the characteristic quantities of generalized Space Syntax. Finally, in section 5 we discuss the criticalities and future developments of the method.

\section{The model}

Basic variables of Space Syntax are the axial lines $v \in V$, which in their more general definition [9] belong to a generic $V$ set. These variables represent a property of the space (the visual lines embraced by the gaze) but are not located in a point. The interaction between two of them $(u, v) \in V \times V=E$ is represented by their intersection: if the corresponding $l_{u}$ and $l_{v}$ visual lines meet, then $l_{u} \cap l_{v} \neq \varnothing$ and $(u, v)=1$, otherwise $\mathrm{f}(u, v)=0$. This property, however, is not defined by $u$ and $v$ variables, but by the inner structure of the city that contains them. In many parts of Manhattan, for example, these lines connect two separate sets of a bipartite graph [8], while in a medieval city the nodes are much more interconnected [9].

In general, consciously or unconsciously, city planners establish a connectivity criterion for these lines [9]. However, variables $v \in V$ are generic variables and may indicate urban properties different from visual lines. So, for instance, nothing precludes us from identifying $v$ with the use of a street, which is pedestrian, driveable, semi-pedestrian, etc.; the variable therefore measures a property of that street. We would then like to use the results and techniques of Space Syntax to understand how the structure of the city affects the interaction between these uses. To do so, we introduce a rigorous process of constructing the corresponding graph.

Considering a generic road graph $G=(V, E)$, where the vertices are the intersections or other road-spots and the edges are their connections, and superimposing it on a map of the city as in Figure 1: if the nodes are sufficiently dense, all the results are insensitive to the choice of this graph.

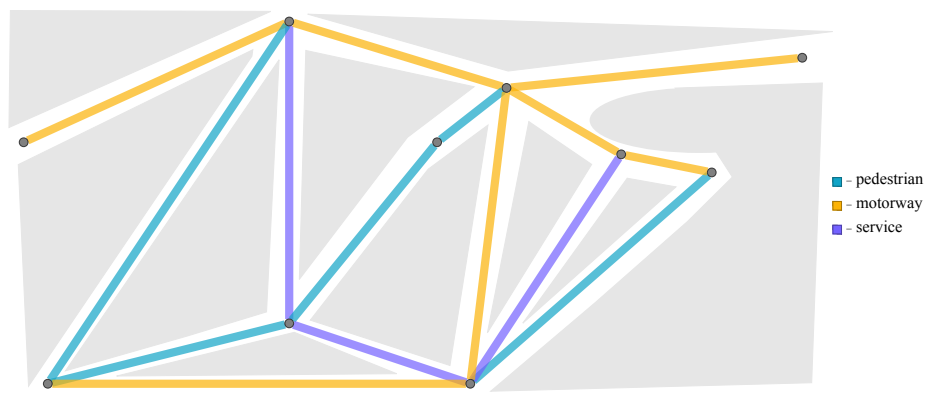

Figure 1. The primary graph $G(V, E)$ for a street network 
Different colors indicate different uses of the streets: pedestrian, driveable and service, while grey areas represent inaccessible spaces. Each color is therefore a property of space. In the particular case of Space Syntax, properties are the axial lines.

From the graph $G$ of Figure 1 we can construct the line graph $L(G)$, the graph whose nodes derive from the edges of $G$. If two nodes of $L(G)$, that is two edges of $G$, have a node in common, then they will be connected in $L(G)$. In substance the edges of $G$ become nodes of $L(G)$, and they are connected only when the two starting edges cross each other. The line graph of Figure 1 is shown in Figure 2.

From graph $G$ of Figure 1 we can construct the line graph $L(G)$, the nodes of which derive from the edges of $G$. If two nodes of $L(G)$, that is two edges of $G$, have a node in common, then they are connected in $L(G)$. In substance, the edges of $G$ become nodes of $L(G)$, and are only connected when the two starting edges cross each other. The line graph of Figure 1 is shown in Figure 2.

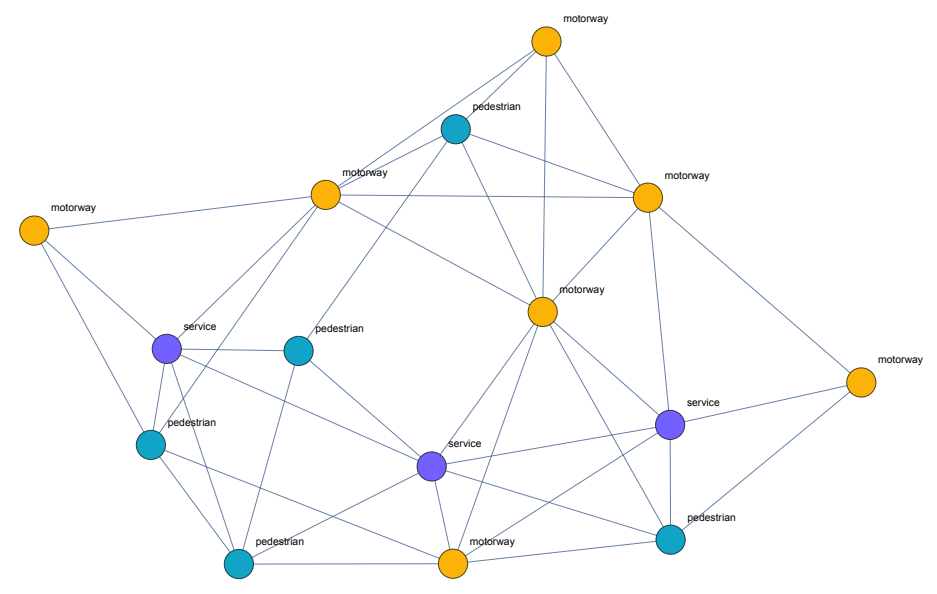

Figure 2. The corresponding line graph $L(G(V, E))$.

We can immediately see that the properties of the $G$ edges become properties of the vertices of $L(G)$, which is a colored (non-proper vertex coloring) non geolocalized graph. Graph $L(G)$ then describes the topology of the city in relation to the property we chose to study.

Next step is to contract the nodes of $L(G)$ that are connected and have the same colour. A set of nodes connected with the same property can be represented by a single node, but only if in the contraction process all the nodes maintain the original connections with the nodes of different colour. We thus obtain the contracted line graph $L_{C}(G)$. The result of our example is given in Figure 3.

The next step is to contract the nodes of $L(G)$ that are connected and have the same color. A set of nodes connected with the same property can be represented by a single node, but only if in the contraction process all the nodes maintain the original connections with the nodes of different color. We thus obtain the contracted line graph $L_{C}(G)$. The result of our example is shown in Figure 3. 


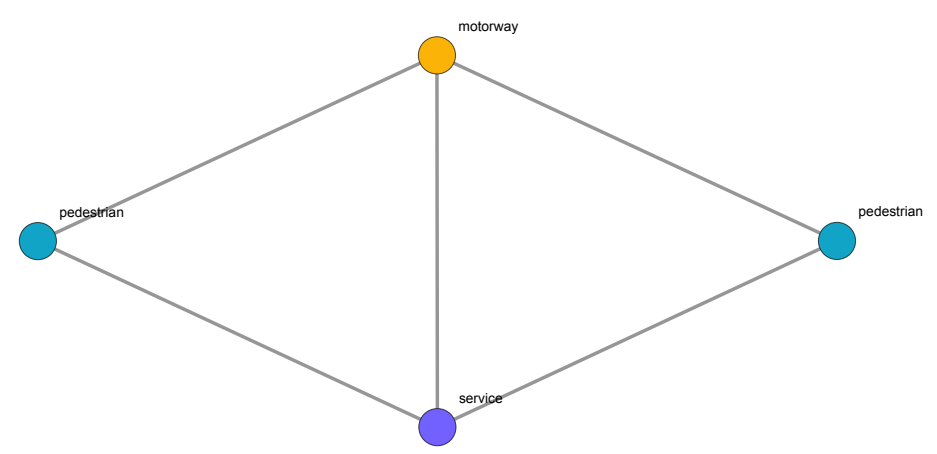

Figure 3. The line graph $L_{C}(G(V, E))$ after contraction of same-color adjacent nodes.

We observe that in Figure 3 pedestrian zones are separated from motorway and service areas, which was also evident when looking at graph $G$. However, when analyzing a large space with thousands of connections, the connective structure is not immediately evident. Moreover, as we see in the following section, in the contracted graph $L_{C}(G)$ it is possible to measure all the parameters of Space Syntax (and not only), such as relative asymmetry, centrality, degree distribution or spectral gap. In this case, however, these measurements do not refer to axial or visual lines, but to the property we have chosen to label the $G$ edges. Finally, we note that if we had chosen visual lines as labels, we would have obtained exactly the same results as with the Space Syntax approach.

\section{The Colosseum case study}

We used the method described in the previous section to compare the current state of the road network in the central archaeological area of Rome with the one planned by the Department of Architecture of Roma Tre University [1]. In its present state, pedestrian access in the area is hindered partly by many structural constraints and partly by some roads that are only accessible to motor vehicles. In particular, via di San Gregorio (the longest red road in Figure 5), the road that extends from the Circus Maximus to the Arch of Constantine, is a road that occupies two carriageways with three lanes, leaving little space for pedestrians. 


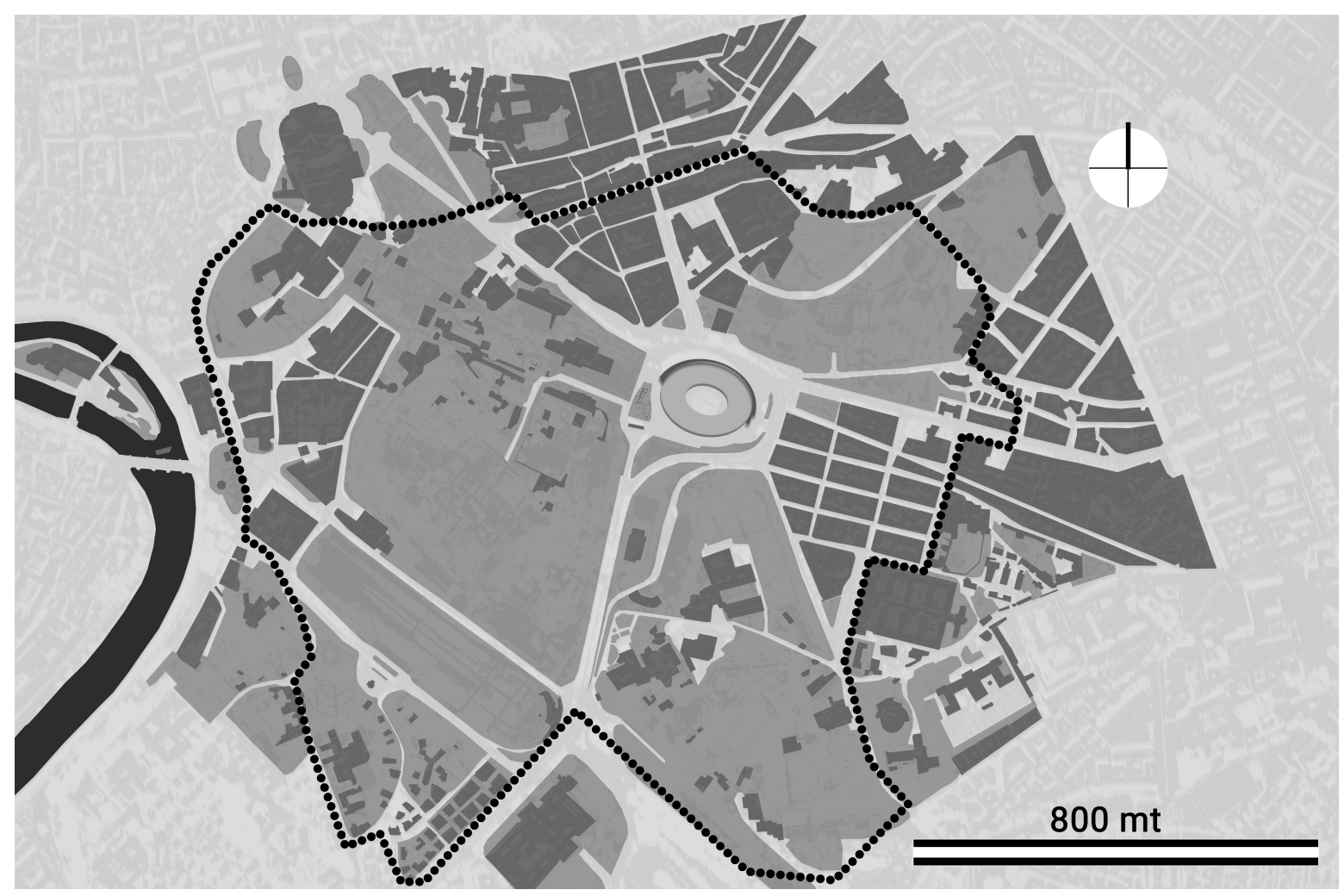

Figure 4. The central archaeological area of Rome (Italy), details of Colosseum area.

The project [1] foresees reducing the carriageway and transforming four of the six lanes into a wide pedestrian avenue that connects the Circus Maximus with the Arch of Constantine and then the Colosseum (Figure 4).
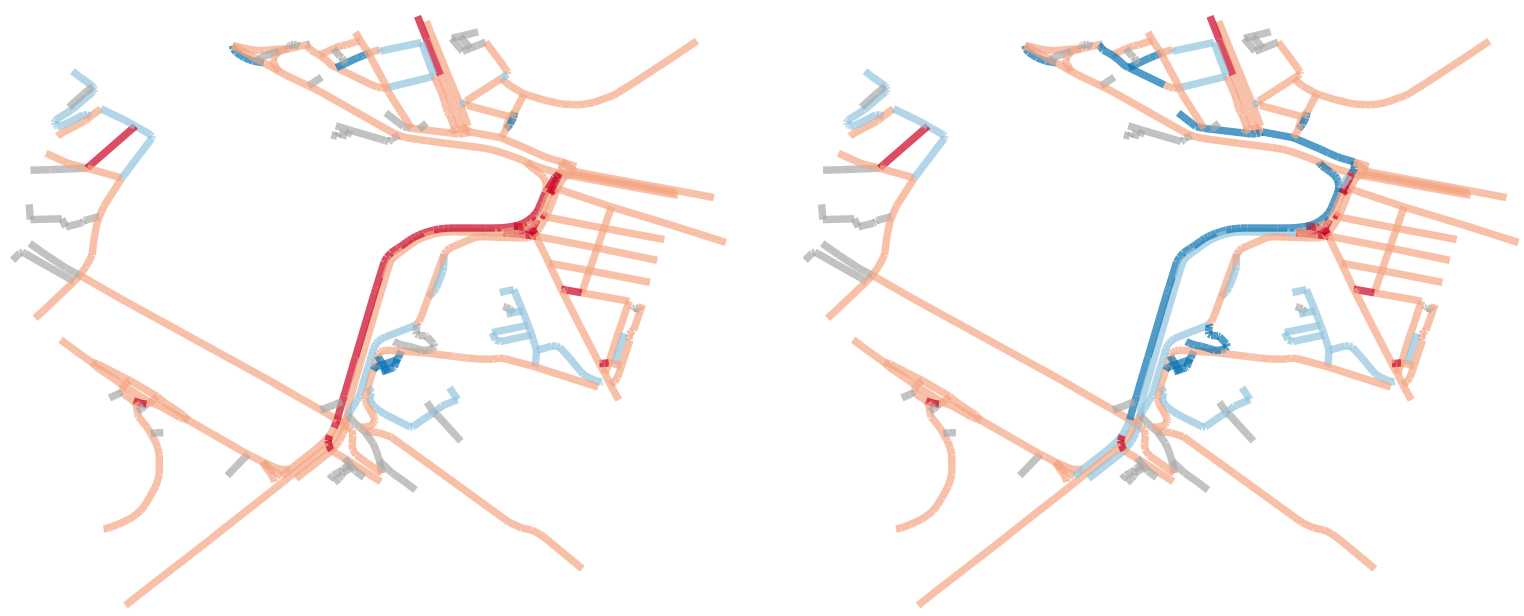

Figure 5. The road network in the central archaeological area as in the current situation (left) and in the project (right). 
The street colors of Figure 5 indicate the use of the road according to the legend in Figure 6 below.

- For vehicles only (i.e. secondary).

$\square$ - Mostly for vehicles (i.e. tertiary).

$\square$ - Service.

$\square$ - Mostly pedestrian (i.e. residential).

—- For pedestrian only.

Figure 6. The variables used in the model.

(1)

The effects of this small change in traffic flow can be analyzed by applying Space Syntax methods to the graph generated by variables that are no longer axial lines, but road uses. Applying the method described in the previous section, we obtain the two corresponding contracted line graphs, where the nodes represent the contraction of the line graph of adjacent roads with the same color (use) as in Figure 6.
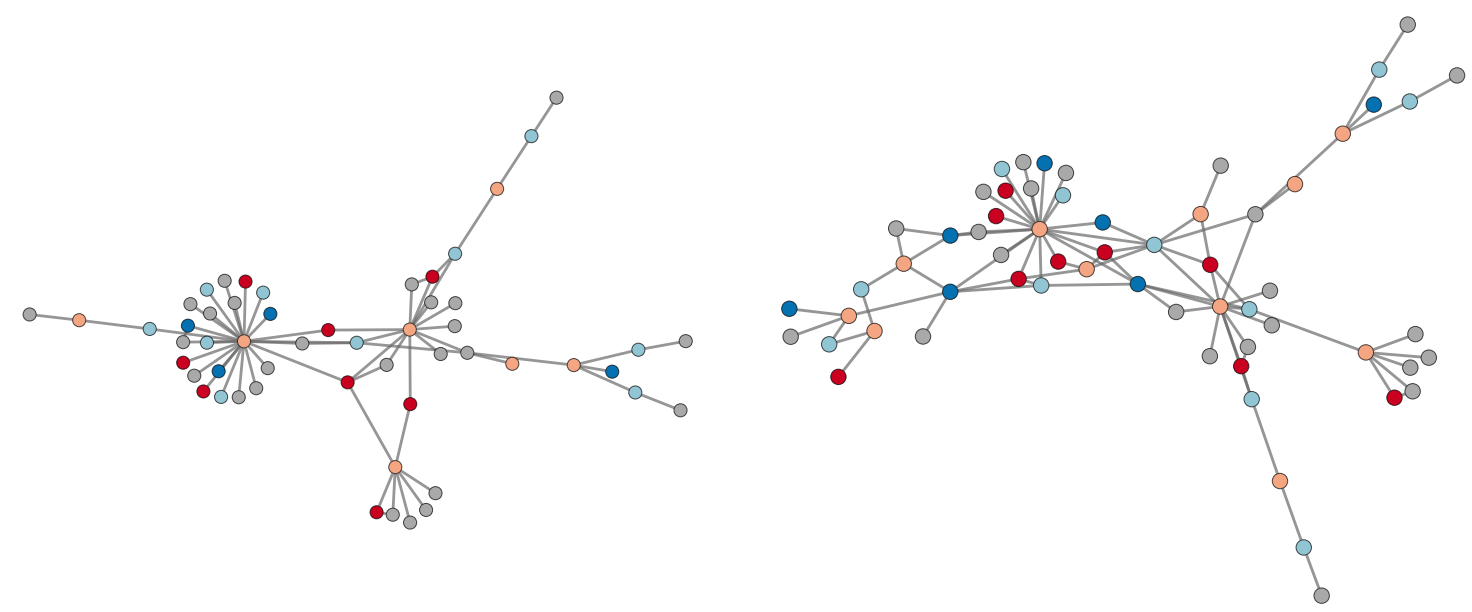

Figure 7. The contracted line graphs $L_{C}(G)$ of the road network in the central archaeological area, as in the current situation (left) and in the project (right).

\section{Results}

We can examine the graph obtained in the previous section with Space Syntax methods, bearing in mind that the measurements do not refer to spaces and their connections, but to road uses and their connections.

The degree distribution shows the first difference between the status quo and the Master plan. Currently there are few hubs connecting different road uses, while in the project some of these hubs are transformed into nodes with a low degree of connectivity, making the graph more entangled and therefore inherently more fluid [biblio]. The hub with 24 edges decreases in degree to 20 and the grid is more connected, as shown in Figure 7. 

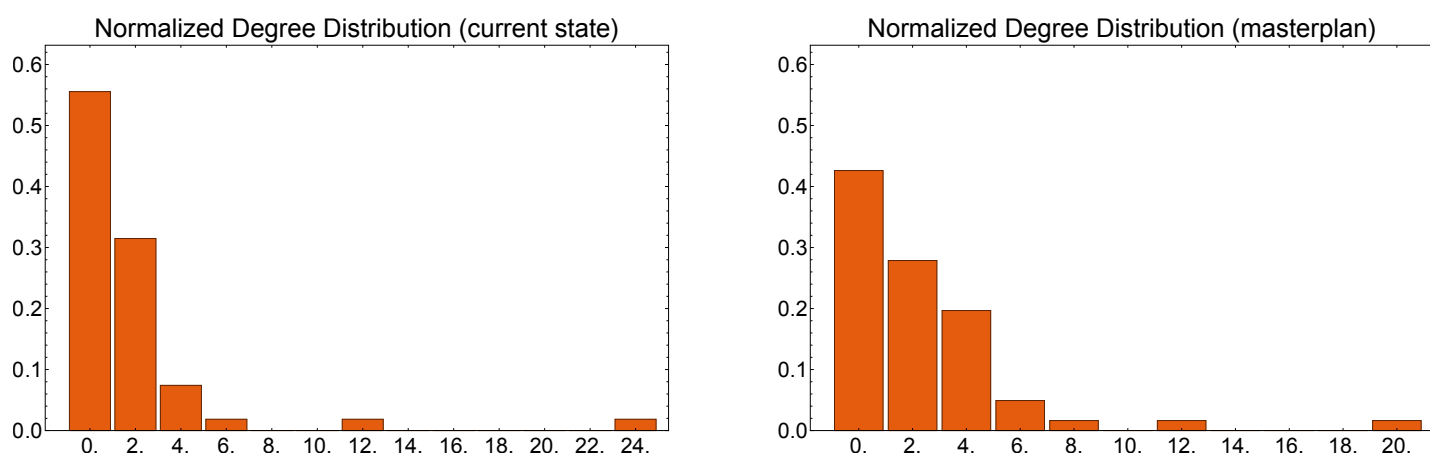

Figure 8. Degree distribution of the central archaeological area, as in the current situation (left) and in the project (right).

Real Relative Asymmetry (RRA) provides a measure of the integration or segregation of the city [10]. In the case of Space Syntax the city integrates or separates the axial lines in a generic sense; in our case it integrates or separates the uses of roads. The distribution of this quantity shows that the histogram of Figure 9 moves a little to the left, towards a topology that is less segregated in the Master plan than in the current situation. The pedestrianization of via di San Gregorio does not in fact eliminate the carriageways, but widens and improves the parallel pedestrian route. This layout makes it possible to maintain the connectivity of motor traffic, but at the same time connects different footpaths and reduces pedestrian segregation.
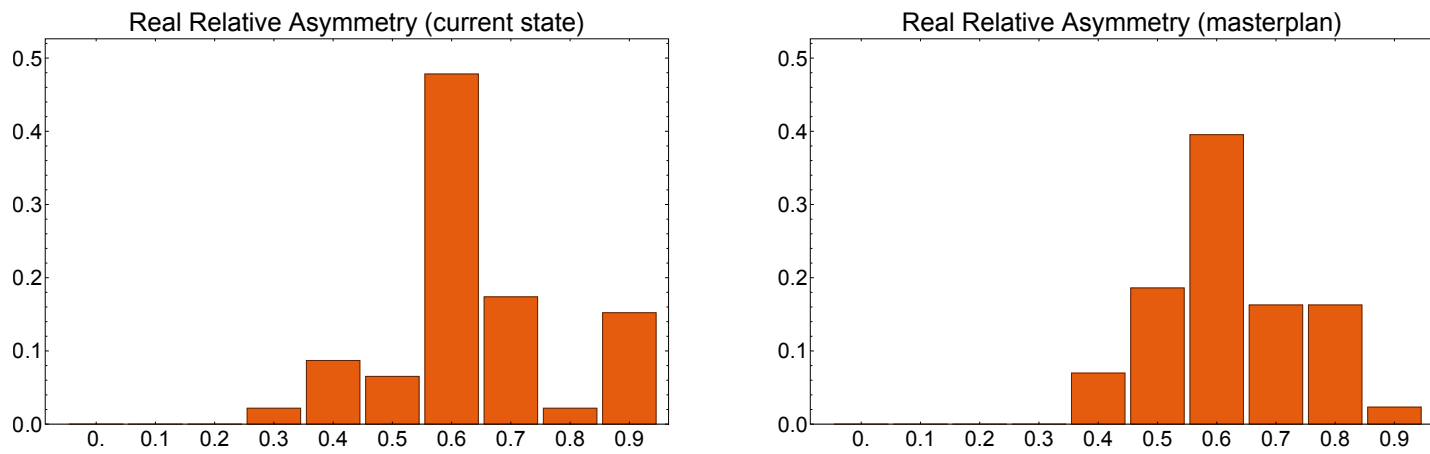

Figure 9. The distribution of Real Relative Asymmetry of the central archaeological area, as in the current situation (left) and in the project (right).

The distribution of Integration [10] clarifies the proposed method and the efficiency of the measures suggested by the Master plan. In our case, contrary to what happens in classic Space Syntax theory, it does not measure the number of (normalized) steps needed to move from one space to another, but the number of different (normalized) road-uses that need to be changed. The difference with respect to the space syntax of the axial lines is not large. In our case, spaces are characterized by the use in terms of mobility and interactions between different uses; in the "classic" case, by their visibility and interaction of views.

In both cases, the information we obtain is related to topological character and we have no information about the size of the spaces or the time it takes to travel the roads. However, metric information can be used as a primary graph label $G(V, E)$ and become an additional parameter of the topological description. From Figure 9, we can see that the Master plan distributes the integration in a more uniform way, moving it towards greater connectivity. 

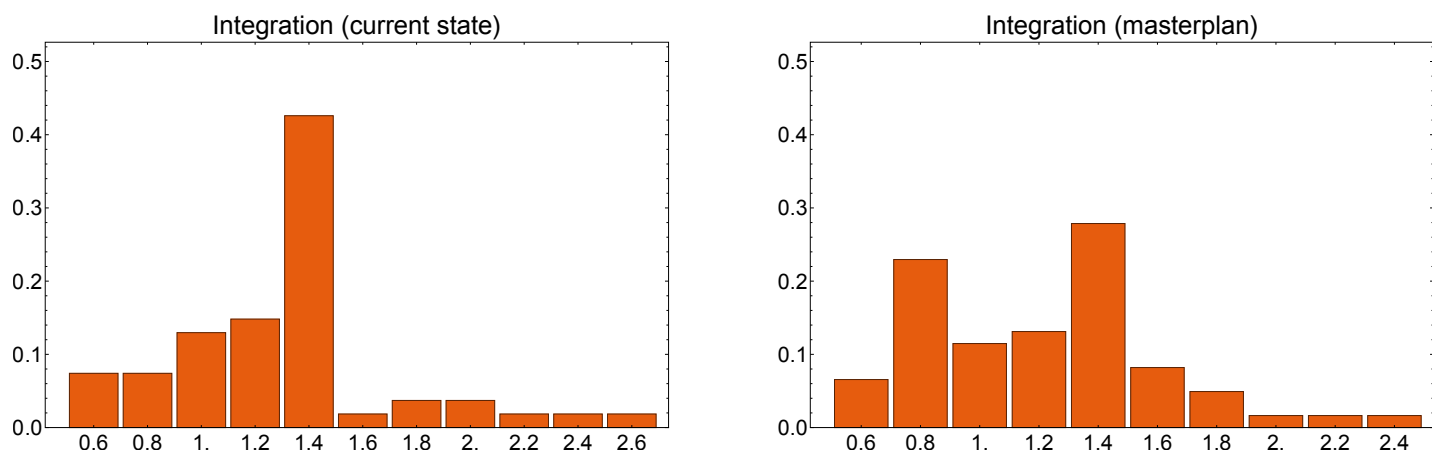

Figure 10. The distribution of Integration of the central archaeological area, as in the current situation (left) and in the project (right).

Finally, in Figure 11, the last measure of Space Syntax we consider is Total Depth, which indicates the sum of depths from a root line to all others, depth meaning the fewest number of changes of lines intervening between the root and another [10]. Here, too, we represent its distribution, because the aim of the Master plan is to make these quantities more uniform. In fact, the plan for via di San Gregorio amplifies the histogram making the probability of crossing a road use, passing from one use to another, more uniform.
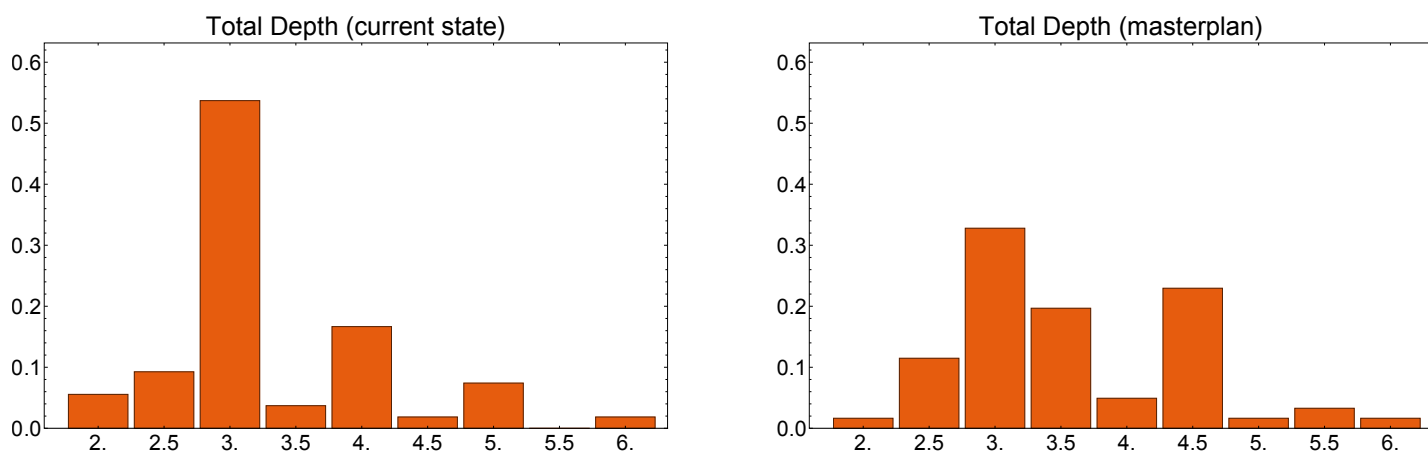

Figure 11. The distribution of Total Depth of the central archaeological area, as in the current situation (left) and in the project (right).

In this section we indicated the urbanistic quantities which are most relevant for evaluating the effects of the Master plan with respect to the status quo, but all the characteristic variables of space syntax can be calculated on the graph of Figure 7.

The tool developed makes it extremely easy to compare qualitatively and quantitatively. Analyzing the project, it is evident that a minor pedestrianization intervention has a very significant effect on the general connectivity of the area and on the interchange between different mobility criteria. However, we underline that as with Space Syntax, the method is not used to design, but only to objectively evaluate the effects of a plan.

\section{Discussion}

We introduced a generalization of Space Syntax to extend the quantities that describe axial lines to generic urban variables. The method is based on the construction of a primary graph whose edges are labeled and categorized by the values of selected variables. The choice of the primary network can be absolutely generic, as long as it is sufficiently dense. For simplicity we chose to identify the edges with road sections, but we could have superimposed any grid on the map of the city. Superimposing a dense square grid or random network on the city and coloring the edges with the values of the corresponding 
variables, we obtain a graph that, once transformed into a line graph and then contracted, always produces the same result.

In fact, it is the city itself that connects urban properties: relationships do not change when we modify the method of measuring them. We also observe that use of the proposed method clarifies some aspects of Space Syntax that are not evident in the original formulation, in particular the statistical nature of many urban quantities. In this sense, greater reliability of the results is obtained when dealing with very large graphs. However, this does not mean that Space Syntax is more reliable for studying a large city than a building, but rather that in the analysis of small spaces it is necessary to superimpose a denser network, or equivalently to measure the quantities that interest us in a very detailed way.

Finally, let us point out that the proposed method makes it possible to compare different variables. For example, we could have measured noise pollution currently and under the Master plan, and compared the corresponding graphs and corresponding quantities of the generalized Space Syntax. The model of interaction between different graphs will be discussed in a later paper.

Author Contributions: Conceptualization and Data curation: R.DA., M.S.; Formal analysis and Investigation: R.DA.; Methodology: R.DA., M.S.; Resources, Supervision, Visualization and Writing - original draft: R.DA.; Writing - review \& editing: MS.

Funding: This Research received no external funding.

Acknowledgments: The authors thank Giovanni Caudo, Giovanni Longobardi, Martina Pietropaoli, Maria Margarita Segarra Lagunes, Nicola Vazzoler, Tiziana Casaburi, Dario Aureli, Mauro Baioni, Milena Farina, Sofia Sebastianelli and all the working group for the Colosseum Masterplan and Isabella Goldmann for her helpful advice. This work was partially supported by MIUR and BIMACT funds ......

Conflicts of Interest: The Authors have no conflicts of interest to declare.

\section{References}

1. Amarilli, F., Aureli, D., Baioni, M., Casaburi, T., Caudo, G., D'Autilia, R., Di Segni, D., Farina, M., Longobardi, G., Pietropaoli, M., Pelusio, B. Sebastianelli, S., Segarra Lagunes, M. M., Vazzoler, N., Piano di assetto dell'area archeologica monumentale del Colosseo, University Roma Tre, 2017.

2. Blanchard, P. and Volchenkov, D. Mathematical Analysis of Urban Spatial Networks.; Springer Science \& Business Media: Berlin, 2008, ISBN-9783540878292

3. Cardillo, A., Scellato, S., Latora, V., et al., Structural properties of planar graphs of urban street patterns., Physical Review E 73: 066107, 2006.

4. Hillier, B. Space is the Machine: A Configurational Theory of Architecture.; Createspace Independent Publishing Platform; pp. 32-58, ISBN-9781511697767.

5. Jiang, B., Claramunt, C., Topological analysis of urban street networks., Environment and Planning B 31: 151-162, 2004.

6. Porta, S., Latora V., Fahui Wang, Rueda S., Strano E., Scellato S., Cardillo A., Belli E., Càrdenas F., Cormenzana B., and Latora L., Street Centrality and the Location of Economic Activities in Barcelona. Urban Studies, Vol. 49, Issue 7, pp. 1471-1488, https:/ / doi.org/10.1177/0042098011422570

7. Stavroulaki, I.,Marcus, L., Berghauser Pont, M., Nilsson,L., C., S., Representations of street networks in space syntax towards flexible maps and multiple graphs, 11th International Space Syntax Symposium, SSS 2017, Lisbon, Portugal, 3-7 July 2017, Vol. 5 p. 174.1-174.16, 2017.

8. Volchenkov, D., Blanchard, P., Random walks along the streets and canals in com-pact cities: Spectral analysis, dynamical modularity, information, and statistical mechanics. Physical Review E 75(2) (2007).

9. D'Autilia, R., Spada, M. Shaping ideal cities: The graph representation of the urban utopia, Environment and Planning B: Urban Analytics and City Science First Published June 29, 2017, https: / / doi.org/10.1177/2399808317716163

10. Hillier, B., Hanson, J., The Social Logic of Space, Cambridge University Press: Cambridge, 1984. 